\title{
Innovation collaborative et innovation ouverte : caractéristiques de leur mise en œuvre
}

\section{Collaborative innovation and open innovation: characteristics of their} implementation

\author{
Camille Aouinaït ${ }^{1}$ \\ ${ }^{1}$ Production fruitière en région alpine, Agroscope, Suisse, camille.aouinait@gmail.com
}

RÉSUMÉ. L'innovation ouverte et l'innovation collaborative sont largement répandues dans différentes disciplines, du management à l'économie en passant par les sciences sociales. Ces concepts ont été développés en opposition à l'innovation fermée qui promeut des activités internes aux firmes, ne s'appuyant que sur les ressources déjà présentes. L'ouverture des frontières des entreprises permet d'intensifier les collaborations avec divers types d'acteurs, pour soutenir l'innovation à l'interne. Par ailleurs, le degré d'ouverture que les entreprises doivent assurer pour promouvoir les processus d'innovation sont sources de divergence dans plusieurs travaux. De plus, peu de travaux ont étudié la dimension territoriale de l'innovation ouverte. La proximité physique serait une condition nécessaire et minimale pour l'innovation, ainsi que d'autres dimensions de la proximité (cognitive et institutionnelle). Les systèmes nationaux d'innovation, districts industriels et clusters favorisant la production et circulation de connaissances sont à l'œuvre et soutenus par des politiques locales, et fleurissent depuis plusieurs décennies. Finalement, l'innovation ouverte favorise une diversité de partenariats qui deviennent possibles, bien que cela engendre aussi des difficultés à différents niveaux entre les entreprises impliquées.

ABSTRACT. Open innovation and collaborative innovation are widely used in a variety of different disciplines, from management to economics and social sciences. These concepts were developed in opposition to closed innovation, which promotes activities that are internal to firms, relying only on the resources already present. Firms opening their borders allows the intensification of collaborations with various types of actors to support innovation internally. The degree of openness that firms must ensure to promote innovation processes is a source of divergence in several works. Furthermore, few works have studied the territorial dimension of open innovation. Physical proximity is a necessary and minimum condition for innovation, alongside other dimensions of proximity (cognitive and institutional). National innovation systems, industrial districts and clusters promoting the production and circulation of knowledge have been at work, supported by local policies and have flourished for several decades. Finally, open innovation promotes a diversity of the partnerships that become possible, although this also creates difficulties at different levels between the involved firms. MOTS-CLÉS. Activités collaboratives, connaissances, degré d'ouverture, droits de propriété intellectuelle, opportunité de marché, territorialité, intermédiaires.

KEYWORDS. Collaborative activities, knowledge, degree of openness, intellectual property rights, market opportunity, territoriality, intermediaries.

\section{Introduction}

L'innovation est un facteur clé de l'économie des connaissances, permettant d'accroître la compétitivité des entreprises par la différenciation et l'utilisation de ressources spécifiques. Les entreprises ne pouvant plus innover seules, les connaissances utiles pour générer des innovations proviennent à la fois de sources internes et externes à l'entreprise [CHE 03].

L'innovation collaborative et l'«open innovation» sont des concepts utilisant l'ouverture sur l'extérieur des frontières de l'entreprise, faisant appel à des échanges avec différents types d'acteurs. La collaboration et l'exploitation de nouvelles sources d'idées sont au coeur de ces notions. Ces termes sont utilisés indifféremment dans la suite de cet article.

L' «open innovation » ou innovation ouverte (IO) est définie par Henri Chesbrough telle qu'un paradigme mis en opposition à l'innovation fermée (voir Figure 1) reflétant un modèle d'intégration verticale traditionnelle, où les activités de Recherche et Développement $(R \& D)$ sont internes à la 
firme ${ }^{1}$. La fermeture des frontières de l'entreprise à son environnement extérieur produit des solutions innovantes qui seront diffusées sur le marché classiquement utilisé [CHE 12].

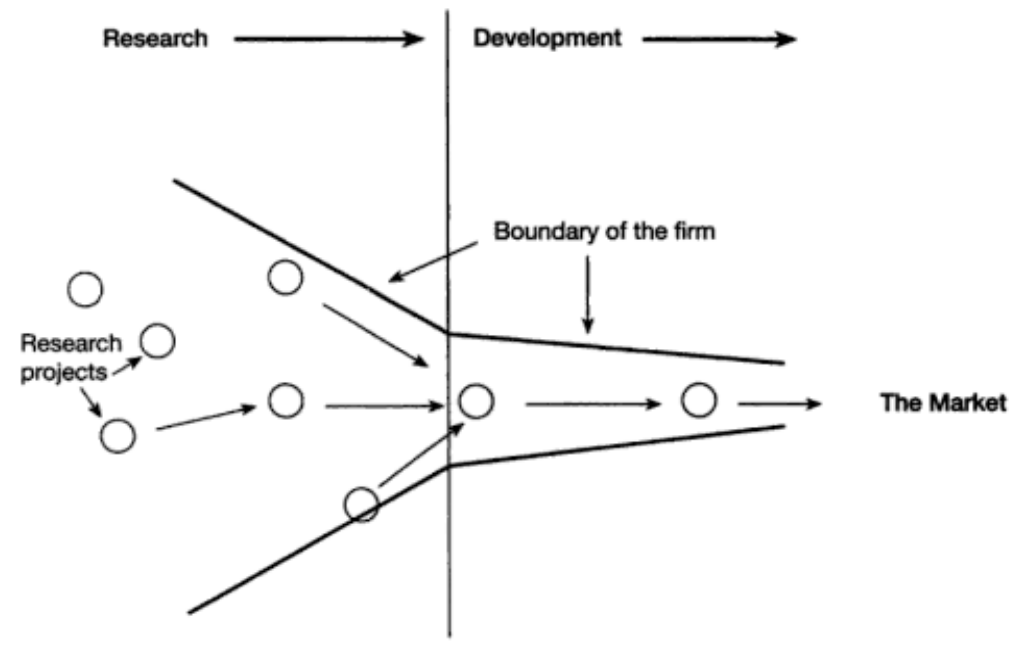

Figure 1. Paradigme de l'innovation fermée défini par Chesbrough [CHE 03]

L'innovation ouverte est un modèle d'innovation collaborative, définie comme "l'utilisation d'entrées et de sorties de savoir pour accélérer l'innovation interne et élargir les marchés de l'utilisation externe de l'innovation respectivement» [CHE 06]. Sur la Figure 2 ci-dessous, les différents flux d'échanges d'informations, de connaissances et autres intrants avec l'externe via des frontières poreuses sont soulignés. De nouvelles sources de technologies peuvent être utilisées à tout moment dans le processus de R\&D. Les résultats sont distribués par les canaux habituels ainsi que sur de nouveaux marchés. Cette nouvelle disposition caractérisant l'innovation ouverte apporte des possibilités inédites de partenariats avec des acteurs ayant des profils variés (par exemple des firmes travaillant sur le même marché, des fournisseurs ou des laboratoires de recherche).

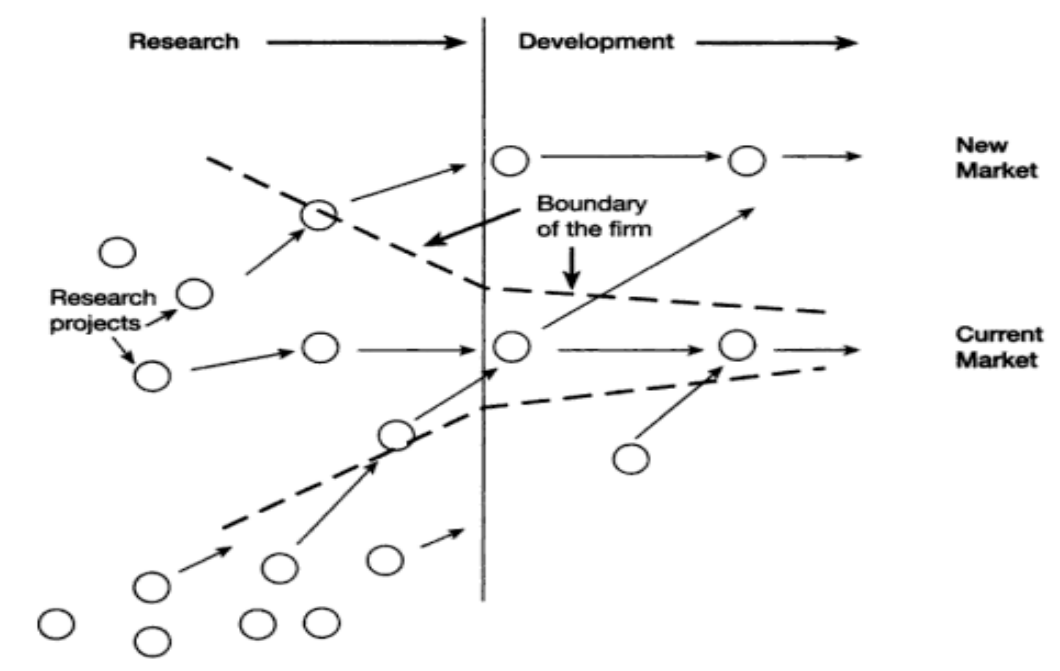

Figure 2. Paradigme de l'innovation ouverte défini par Chesbrough [CHE 03]

\footnotetext{
${ }^{1}$ L'intégration verticale opposée à l'intégration horizontale, est mode de management des activités qu'une entreprise réalise. En intégrant les différents processus tels que la production, la transformation, la commercialisation de produits, la firme peut voir son rendement global ou différencié par activités, ainsi que ses coûts concernant divers postes de dépenses optimisés. Certains secteurs sont plus marqués par l'intégration verticale que d'autres, comme le secteur agroalimentaire sur le continent américain. L'industrie des fruits et légumes ou des produits laitiers est traditionnellement intégrée verticalement, a contrario du secteur des céréales.
}

DUFEU A., Analyse des processus d'intégration-désintégration verticales. Cahier de recherche no. CR02, 2011. 
Dans une première section, les concepts directeurs et les notions de base associés à l'innovation ouverte et collaborative sont présentés. Un bref historique ainsi que les principales occurrences dans la littérature de différents domaines sont ensuite présentés. Les problématiques inhérentes à ces concepts viennent apporter des compléments, notamment les types de partenariats possibles dans un tel contexte, l'importance des proximités pour l'innovation, les acteurs à l'œuvre et les difficultés engendrées. La dernière section expose les conclusions et ouverture.

\section{Retour sur la notion d'innovation ouverte et innovation collaborative : définition et implications}

\subsection{Historique}

L'innovation ouverte ou « open innovation » est un terme apparu en 2003 dans les travaux d'Henri Chesbrough, notamment à travers l'ouvrage intitulé Open Innovation: The New Imperative for Creating and Profiting from Technology (Harvard Business School Press).

Le terme «d'open innovation» a émergé en parallèle au terme de «l'open source » qui était appliqué aux logiciels et avait connu du succès de par les changements apportés dans le domaine ${ }^{2}$. Cela a facilité la diffusion du modèle d'Henri Chesbrough. Ce dernier a fourni des modifications notables quant aux Droits de Propriété Intellectuelle (DPI) utilisés jusqu'alors [LOI 11]3.

Selon Chesbrough, plusieurs facteurs d'érosion ont contribué au déclin du modèle " fermé ». Le premier concerne la mobilité et disponibilité des travailleurs qualifiés. Le second quant à lui se rapporte au marché du capital-risque, où les entreprises spécialisées diffusent les innovations par l'élaboration de spin-off en particulier. Un autre facteur d'érosion concerne les nouvelles capacités des fournisseurs à réaliser des activités de R\&D. Les connaissances et autres intrants utiles à l'innovation sont redistribués différemment entre les acteurs [ISC 11].

Depuis sa création, le terme d'innovation ouverte a été indiscutablement utilisé. Chesbrough a recensé 483 millions de liens en cherchant «Open innovation » dans le moteur de recherche de Google [CHE 12]. Après son premier livre sur l'Open Innovation en 2003, l'auteur a porté son attention sur les Open Business Models en 2006. Selon lui, «le modèle d'entreprise joue un rôle essentiel dans le processus d'innovation ». Le secteur des services, qui est très rémunérateur pour les entreprises, a ensuite été étudié selon un angle d'innovation ouverte. Les firmes peuvent bénéficier de retombées positives de par la mise en relation de divers acteurs pour résoudre des problèmes et apporter des solutions grâce à la diversité des compétences et connaissances de chacun. Dans le cas de la firme Xerox et son centre de recherche à Palo Alto (Palo Alto Research Center), de nombreux projets initiés à moyen et long terme ont échoué car le modèle commercial d'alors n'était pas adapté et l'entreprise n'a pas pu obtenir la vraie valeur de l'innovation créée.

\footnotetext{
${ }^{2}$ Un logiciel dit « open source » est un logiciel qui est libre d'utilisation pour tous. Ce type de logiciel est développé par des communautés d'utilisateurs pour ces mêmes utilisateurs. Depuis leur création dans les années 1960-1970, des milliers de projets et de nombreuses communautés ont été développés à travers le monde.

VON HIPPEL E., «Innovation by User Communities: Learning from Open-Source Software», MIT Sloan Management Review, 82-86, 2001.

VON HIPPEL E., « Open Source Software and the "Private-Collective” Innovation Model: Issues for Organization Science », Organization Science, 14 (2)208-223, 2003.

${ }^{3}$ Les deux principaux buts des droits de propriété intellectuelle (DPI) sont de protéger l'innovateur de l'utilisation frauduleuse de l'innovation et de conférer un droit exclusif temporaire sur l'innovation en général de 20 ans en France et 17 ans aux États-Unis par exemple. Des revenus peuvent être tirés de ces DPI. Le contrôle de la diffusion et de la commercialisation des informations relatives au produit protégé revient à l'innovateur. Le brevet est le DPI le plus traité dans la littérature. Il existe aussi les licences, les droits d'auteur ou encore les marques. LIOTARD I., «Les droits de propriété intellectuelle, une nouvelle arme stratégique des firmes», Revue d'économie industrielle, 89(1), 69-84, 1999.
} 


\subsection{Innovation collaborative et « écosystèmes d'innovation »}

L'innovation ouverte provient du domaine du management de l'innovation et de la technologie et concernait principalement la Recherche et Développement. Désormais, les domaines des stratégies d'entreprises et comportement organisationnel s'y intéressent, mettant en avant l'implication croissante de cette théorie dans les diverses voies des sciences sociales notamment (petites et grandes firmes, high-tech et low-tech) [GAS 10].

D'après Isckia et Lescop, le domaine des écosystèmes des affaires est proche de l'innovation ouverte. La diffusion de l'innovation et le rôle central des entreprises dans un écosystème défini sont des fers de lance de ce champ de recherche, avec pour but essentiel la création de valeur [ISC 11].

En outre, l'innovation collaborative autorise la génération de projets innovants [LOI 11]. La collaboration permet de rentrer dans des logiques de co-développement entre divers acteurs ayant des intérêts communs [ISC 11]. La R\&D est un poste économique et technologique où l'organisation des collaborations est stratégique. L'ouverture des entreprises dans le processus d'innovation devient alors primordiale.

Par ailleurs, les externalités et impacts de l'innovation ouverte sont répartis à l'intérieur et l'extérieur des frontières de l'entreprise. L'innovation ouverte peut être réalisée selon deux processus : inside-out et outside-in. Le premier processus requiert que les firmes permettent aux idées inutilisées ou peu utilisées de sortir des frontières de la firme pour une utilisation externe par d'autres acteurs. Le second fait référence à l'ouverture des processus d'innovation des firmes à divers intrants et contributions externes [CHE 12, ISC 11]. Par conséquent, les nouvelles idées peuvent trouver leurs sources autant à l'intérieur qu'à l'extérieur de la firme, en participant aux activités de R\&D ou en fournissant d'importantes ressources par exemple. L'innovation collaborative permet de réaliser un « sourçage », d'aller chercher des sources d'innovation provenant de l'extérieur de la firme. Ceci souligne l'importance du recours à divers mécanismes de recherches d'informations et de connaissances pour pouvoir innover. L'usage du réseau dans lequel les entreprises sont intégrées est alors primordial pour enrichir leur base de connaissances et développer des collaborations avec divers partenaires (par exemple autres entreprises, universités, recherche, associations).

De plus, les pôles de compétitivité sont un regroupement sur un territoire identifié d'acteurs publics et privés tels que des petites, moyennes et grandes entreprises, organismes de formation et centres de recherche, travaillant sur une thématique commune. Les pouvoirs publics les soutiennent financièrement. Ces pôles visent à promouvoir l'innovation, à développer des projets collaboratifs et favoriser la croissance dans le territoire. Dans les pôles de compétitivité utilisant 1'IO, l'optimisation des capacités d'innovation sur un plan collectif est un des buts recherchés [LER 14]. Le délai entre la création d'une innovation et sa mise sur le marché peut être réduit. Ce processus est ainsi bénéfique pour les entreprises collaborant dans ces actions collectives.

L'un des avantages de l'innovation collaborative repose sur le fait que l'entreprise s'ouvre à son environnement extérieur et devient plus visible pour des acteurs qui pourraient être intéressés de collaborer et gagner des connaissances et compétences spécifiques requis dans des projets innovants [LOI 11]. Cependant, ce modèle d'innovation peut jouer un rôle sur les stratégies définies de l'entreprise au niveau des relations établies ou à développer avec des partenaires externes dans le but d'acquérir des compétences et connaissances et des modalités de propriété intellectuelle (par exemple à quel degré les connaissances circulantes de manière ouverte sont libres d'utilisation) [LER 14]. 
Enfin, malgré l'ouverture des entreprises vers leur environnement extérieur, celles-ci doivent pratiquer de la R\&D en interne à un niveau minimum, non défini par les études sur le sujet, pour qu'elles puissent travailler leur capacité d'absorption [CHE 12, LOI 11]. En effet, pour exploiter les ressources et connaissances recueillies à l'extérieur, notamment à travers des collaborations établies, les firmes doivent pouvoir les réutiliser en interne_[LOI 11]4.

\subsection{L'importance de l'environnement pour la mise en œuvre de stratégies d'innovation collaborative et les risques encourus}

L'innovation ouverte fait appel à des acteurs et entreprises pouvant apporter des ressources à l'interne de l'entreprise. Des flux de l'intérieur vers l'extérieur de l'entreprise ont également lieu. Tous ces mouvements de produits et connaissances se font dans un environnement particulier, que l'entreprise se doit d'avoir analysé. En effet, selon Mintzberg, l'environnement est caractérisé selon la stabilité/dynamique, la complexité, la diversité des marchés et l'hostilité [MIN 82]. Le premier fait référence aux changements occasionnés dans l'environnement le rendant dynamique de par les nouveautés engendrées (par exemple le secteur des nouvelles technologies de communication). Le second comprend le niveau de compétences requis pour acquérir de nouvelles connaissances et les utiliser de manière fréquente (par exemple le secteur aéronautique requiert des connaissances et compétences complexes qui, une fois acquises, peut être intégrées dans des innovations utilisées quotidiennement). La diversité des marchés prend en compte les besoins des clients, les méthodes de distribution et la capacité à se diversifier. Enfin, ce que Mintzberg entend par hostilité englobe le milieu socio-politique dans lequel l'entreprise évolue. La gouvernance des marchés et leur réglementation peut être plus ou moins hostile en fonction du secteur (par exemple le secteur des industries pétrolières bénéficie d'un environnement hostile de par la forte concurrence et tension au niveau des prix et des acteurs en jeu). Toutes ces variables définissant un environnement doivent être prises en compte par les entreprises pour mener à bien leur stratégie d'innovation [STE 14].

\section{Types d'innovation et acteurs impliqués}

\subsection{Innovation ouverte versus innovation fermée}

L'innovation ouverte se situe dans différentes problématiques aujourd'hui. En effet, la dichotomie innovation ouverte vs. innovation fermée est critiquée dans beaucoup de travaux. Elle provient notamment de la différence des frontières permises (poreuses ou non) [DAH 10, ISC 11, LOI 11]. Un modèle d'innovation n'est ni totalement ouvert ni totalement fermé. Des formes hybrides à la croisée des deux modèles d'innovation peuvent exister, telles que Loiler et Tellier le décrivent dans leur article. Il s'agit d'un «modèle intermédiaire alliant les aspects public et privé utilisés dans le modèle ouvert de l'Open Source (« libre participation des utilisateurs ») et le modèle fermé des entreprises traditionnelles («appropriation par la firme des résultats») respectivement. Ce modèle n'est cependant que peu établi car la contrepartie financière est à la base de beaucoup de stratégies d'entreprises où l'innovateur peut « capturer la rente issue de l'innovation » selon le modèle d'appropriabilité choisit.

\footnotetext{
${ }^{4}$ Cohen and Levinthal ont défini la capacité d'absorption comme la «capacité à reconnaître la valeur de nouvelles connaissances, externes, à les assimiler et à les appliquer à des fins commerciales. ». Cette capacité des firmes se construit sur le long terme en se créant une base de connaissances et des compétences internes à la firme, nécessaires pour intégrer les nouvelles connaissances qui sont acquises au fil des projets, notamment à travers la Recherche et Développement. Cette capacité d'absorption des firmes a tendance à être construite sur la capacité d'absorption individuelle des membres des firmes, à être cumulative et dépend de la trajectoire antérieure choisie (par exemple, les choix technologiques et stratégiques que la firme met en œuvre). COHEN W.M., Levinthal D.A., « Absorptive Capacity: A New Perspective on Learning and Innovation », Administrative Science Quaterly, 35(1), 128-152, 1990.

LANE P-J., LUBATKIN M., « Relative absorptive capacity and interorganizational learning », Strategic Management Journal, 19(5), 461-477, 1998.
} 
En outre, l'innovation ouverte implique une co-création de connaissances et innovations ainsi que le transfert des connaissances. Or, il a été largement étudié que le transfert de connaissances peut prendre plusieurs formes selon le type de connaissances concernées: formalisées/codifiées ou tacites/non codifiées ${ }^{5}$. Les connaissances codifiées sont facilement transférables dans divers contextes car non dépendantes des agents réalisant ce transfert. Elles sont généralement enregistrées ou écrites. Les connaissances tacites, quant à elles, sont «sticky », dépendantes du contexte dans lequel elles ont été créées et/ou utilisées et inhérentes aux individus [VON 94].

Une autre critique porte sur l'aspect nouveau du modèle [LOI 11]. En effet, il n'est pas réellement innovant d'avoir recours à des innovations venant de l'extérieur pour en faire profiter les entreprises, comme dans le cas de l'acquisition de licences. Les Droits de Propriété Intellectuelle (DPI) représentent des «dispositifs contractuels » permettant aux firmes de profiter de multiples formes de collaborations [ISC 11]. En effet, l'innovation ouverte, de par sa structure faisant appel à des acteurs externes, permet de redistribuer les coûts du processus d'innovation. D'un côté, les coûts de R\&D peuvent être moindres. D'un autre côté, l'utilisation de DPI permet de générer des revenus. Néanmoins, il est estimé que seulement $20 \%$ des brevets génèrent $80 \%$ des revenus. Tous les DPI ne sont, de ce fait, non propices à une utilisation en interne. Isckia et Lescop considèrent donc que l'avantage de l'innovation ouverte serait de «valoriser ces brevets « dormants » en les proposant à d'autres entreprises » [ISC 11].

Par ailleurs, une autre limite ou frontière du modèle de l'innovation ouverte concerne la mobilité du travail qui est un facteur indispensable pour assurer l'efficience des transferts de projets entre entreprises. Si le personnel change de firme avec un projet, il pourra augmenter l'intégration de toutes les données importantes du projet dans le nouvel environnement [CHE 12]. On peut retrouver ici les facteurs d'érosion discutés par Chesbrough.

Le degré d'ouverture du modèle est questionné dans la littérature. Le degré d'ouverture de l'innovation ouverte demeure restreint, dans le sens où la logique de propriétaire reste de mise. En effet, contre compensation financière, une firme peut céder ses innovations via des licences par exemple (principe d'inside-out ou sortie d'innovation) ou en acquérir (principe d'outside-in ou entrée d'innovation). A contrario du modèle de l'open source pour les logiciels où l'éditeur d'un code source cède ses droits de ce code, pour une modification, amélioration et utilisation conjointe par d'autres utilisateurs. L'ouverture de ce type de modèle est donc plus largement appuyée [CHEI 12, LOI 11].

L'innovation ouverte et l'innovation collaborative sont de ce fait similaires jusqu'à un certain niveau d'ouverture du modèle. Les collaborations possibles et nécessaires pour développer et accélérer des activités innovantes internes aux firmes peuvent être limitées en termes de droits de propriété et des conditions prédéfinies par les acteurs (par exemple type de collaborations contractuelles, type de partenaires et de partenariats).

Par ailleurs, les innovations de type radicales pourraient être développées par le biais du paradigme de l'innovation ouverte d'après Sachwald. Ces innovations radicales peuvent avoir recours à des partenariats pour les phases de recherche, production et commercialisation de l'innovation. Le degré

\footnotetext{
${ }^{5}$ Les connaissances tacites sont dites des connaissances non codifiées, acquises par l'adoption informelle de comportements et de procédures antérieurement appris. Ces connaissances sont généralement ancrées dans les individus et non transmises de manière formelle. Par conséquent, ce type de connaissances est plus difficile à transmettre que les connaissances codifiées telles que les informations documentées et écrites (brevets, certificats, base de données, archives). Ces connaissances explicites sont éditées dans un langage formel et facilement transmissibles.
}

COWAN R., DAVID P.A., FORAY D., « The Explicit Economics of Knowledge Codification and Tacitness », Industrial and Corporate Change, 9(2), 211-253, 1999.

HoweELS J., « Tacit knowledge », Technology Analysis \& Strategic Management, 8(2), 91-106, 1996. 
d'ouverture est donc important à prendre en compte en fonction du type d'innovation souhaitée [SAC 08]. Finalement, les innovations radicales ont besoin de plus d'engagement à long terme de la part des acteurs impliqués dans leur création, ainsi que plus de connaissances, ce qui engendre des partenariats de différentes nature [AGO 13].

\subsection{Rôle de la proximité, exemples avec les clusters et systèmes locaux d'innovation}

La proximité géographique est sujette à produire des externalités qui favorisent l'innovation. Les connaissances tacites, dites informelles, circulent dans un espace géographique limité de par les contacts en face-à-face des agents [PEC 02]. Cette proximité géographique se retrouve dans des écosystèmes créés tels que les clusters et systèmes locaux d'innovation.

La transmission des connaissances est considérée comme plus facile dans un territoire où les activités économiques sont géographiquement proches. Cette territorialisation des activités repose sur une proximité physique importante, où les interactions entre acteurs sont soutenues et facilitées. Cependant, la proximité physique n'est pas toujours garante de succès. Les proximités cognitive et institutionnelle ont aussi leur importance. La première fait référence à une proximité entre acteurs basée sur une base de connaissances proches sur laquelle les recherches d'innovations, de technologies ou autres éléments importants se font [BOS 05]. La capacité des acteurs à absorber de nouvelles connaissances nécessite une proximité cognitive de façon à pouvoir les transmettre et les intégrer. La proximité institutionnelle reflète les caractéristiques communes que des institutions peuvent avoir, facilitant la compréhension de leur environnement et de ce fait, de certains processus mis en œuvre de manière commune. Ces trois types de proximités permettent d'améliorer et augmenter les interactions et la coordination entre acteurs, favorisant l'innovation [BOS 05].

Alfred Marshall, dès 1890, établit le concept de districts industriels, représentant « généralement l'agrégation d'un grand nombre de petits ateliers, comme la création de quelques grandes usines, permet d'atteindre les avantages de production à grande échelle [...] Il est possible de couper le processus de production en plusieurs segments, chacun pouvant être réalisé avec le maximum d'économies dans un petit établissement formant ainsi un district composé d'un nombre important de petits établissements semblables spécialisés pour réaliser une étape particulière du processus de production » [DAU 07].

Michael Porter, quant à lui, définit une notion proche, celle de cluster, comme étant une « concentration géographique d'entreprises interdépendantes : fournisseurs de biens et de services dans des branches industrielles proches ; les firmes livrant le produit final coopèrent avec les universités, et leurs concurrentes » [POR 90]. Le cluster est une «grappe d'entreprises » opérant souvent dans un même secteur d'activités.

Les clusters sont à la base de politiques publiques territorialisées, mettant l'accent sur des caractéristiques précises des territoires et pouvant être axées sur des thématiques définies. Aux ÉtatsUnis, la Silicon Valley est un exemple connu de la réussite de la territorialisation d'entreprises dans un espace donné, spécialisées dans les hautes technologies et l'électronique. En Europe ces politiques sont très répandues. En France, les Systèmes Locaux de Production et les pôles de compétitivité en sont des résultats probants. Dans les années 1980, beaucoup de pays européens ont créé et mis en application des politiques de clusters. La Catalogne et le Pays Basque espagnol sont des exemples revenant souvent dans les études menées sur ce sujet [INS 08]. Dans cette dernière région, l'innovation des filières traditionnelles telles que l'automobile, les industries portuaires, l'électronique ou encore l'énergie a été mise en avant par l'expérimentation des politiques de clusters. En Allemagne, au Danemark avec la Medicon Valley dans la région transfrontalière d'Öresund, clusters de biotechnologies et de produits pharmaceutiques, au Royaume-Uni, ou encore en Italie avec par exemple Turin et les technologies sans fil, la Vénétie et les nanotechnologies, la Lombardie et les biotechnologies ou Bologne et la mécanique sont autant d'exemples de succès de mise en place de clusters [INS 08, RAL 04]. 
La notion a du succès car elle favorise la diffusion des connaissances et s'inscrit dans les externalités de réseaux, où l'utilité pour un acteur est « liée à la présence importante et croissante » des autres acteurs présents. De plus, comme le concept d'innovation ouverte, les clusters sont voués à entretenir des échanges avec l'extérieur. Ceci permet aux entreprises du cluster de bénéficier des retombées locales générées par des partenariats avec des acteurs externes [RAL 04]. Par ailleurs, les clusters sont caractérisés par des économies d'échelle à travers des coûts de transports réduits, les économies d'approvisionnement (par exemple en matières premières ou énergie) et la mutualisation de services [ICHA 08, RAL 04, TOR 16].

A un niveau supérieur, un système national d'innovation peut être défini comme l'ensemble des institutions qui contribuent conjointement ou individuellement à la création, diffusion et utilisation de nouvelles technologies. Des politiques publiques axées sur les progrès technologiques sont conçues et soutenues par ce système, qui est également décliné en système régional ou local d'innovation [WAN 11]. L'ouverture des entreprises en termes de processus d'innovation les amènent à collaborer avec des acteurs locaux, mais également internationaux. Cette internationalisation de l'innovation ouverte permet aux entreprises de renforcer leurs compétences, qui peuvent être manquantes au niveau local. Des laboratoires de recherche peuvent chercher des partenariats avec d'autres acteurs à l'international où les connaissances sont présentes [SAC 08]. Les réseaux qui sont ainsi construits peuvent servir dans différents projets et ainsi produire de l'innovation, avec notamment des retombées économiques au niveau du territoire.

\subsection{Les intermédiaires et leurs rôles dans les processus d'innovation}

Howells et al. définissent les «intermédiaires d'innovation » comme une « organisation ou un organisme qui agit comme agent ou courtier dans n'importe quel aspect du processus d'innovation entre deux ou plusieurs parties » [HOW 06]. Les activités menées par ces intermédiaires peuvent comprendre la recherche d'informations, le traitement des informations et des connaissances, la diffusion des connaissances à la société, la commercialisation de produits, l'évaluation de résultats, un soutien juridique, l'aide dans la recherche de financements et d'autres types de soutien quant à des objectifs d'innovation entre autres. Par ailleurs, les flux d'informations et de connaissances peuvent aussi être enrichis par des boucles de rétroactions entre les acteurs [AOU 19, HOW 06 ].

Les intermédiaires peuvent provenir du secteur public comme du secteur privé. Dans le cas allemand de petites et moyennes entreprises mettant en place des innovations d'éco-efficacité, les entreprises ont un faible niveau d'absorption des connaissances. Avoir recourt à des intermédiaires publics et privés permet de combiner les services que ceux-ci peuvent fournir ; un soutien politique local pour les premiers et des conseils spécifiques et exclusifs pour les seconds [KLE 12].

Selon Agogué et al., quatre principales fonctions sont remplies par les intermédiaires dans un contexte d'innovation. Ils connectent les acteurs entre eux, ils impliquent, engagent et mobilisent les acteurs. Ils ont également un rôle de résoudre, éviter et miter les conflits d'intérêts, et enfin ils stimulent activement le processus d'innovation et les résultats de ce processus [AGO 13]. La spécialisation des intermédiaires de l'innovation peut être engendrée par les besoins des clients. En effet, le recours à plusieurs types de prestataire et prestations associées permet une efficacité dans le traitement des tâches requises. Par ailleurs, les acteurs ayant besoin de ces intermédiaires sont les mieux placés pour savoir quelle expertise leur est manquante et nécessaire dans leurs projets [PON 02]. Une combinaison entre ressources internes et externes est souvent réalisée par les entreprises pour valoriser d'une part les ressources internes et d'autre part assurer l'exploitation et l'exploration efficaces et durables des sources externes [AOU 19]. 


\section{Innovation collaborative : une diversité de partenariats résultante}

Les partenariats engagés au long du cycle de processus de l'innovation sont de différents types. Sur la figure 3, les modes d'innovation selon l'Association européenne de recherche industrielle sont présentés à travers deux axes : la technologie et les marchés de l'entreprise. Le développement interne et l'acquisition sont des actions menées lorsque l'entreprise possède ou veut posséder des technologies et accéder à des marchés stratégiques. A contrario, la vente ou l'essaimage externe sont privilégiés dans des situations peu familières. L'utilisation de partenariats est plus avisée dans ces cas, tout comme sur des marchés non stratégiques, où l'utilisation de compétences et connaissances manquantes à l'entreprise est primordiale pour le bon développement de l'innovation en question.

\begin{tabular}{|c|c|c|c|}
\hline Peu familier & $\begin{array}{l}\text { Coentreprise } \\
\text { (Joint venture) } \\
\text { R\&D sous contrat }\end{array}$ & $\begin{array}{l}\text { Capital-risque } \\
\text { Fond de capital interne } \\
\text { (Internal venture fund) }\end{array}$ & $\begin{array}{c}\text { Essaimage externe } \\
\text { Vente }\end{array}$ \\
\hline $\begin{array}{l}\text { Non } \\
\text { stratégique }\end{array}$ & $\begin{array}{l}\text { Développe me nt conjoint } \\
\text { Acquisition }\end{array}$ & $\begin{array}{l}\text { cession de licences } \\
\text { Prise de participation }\end{array}$ & $\begin{array}{l}\text { Capital-risque } \\
\text { Fond de capital interne } \\
\text { (Internal venture fund) }\end{array}$ \\
\hline \multirow[t]{2}{*}{ Stratégique } & $\begin{array}{c}\text { Acquisition } \\
\text { Développement interne }\end{array}$ & $\begin{array}{l}\text { Développement interne } \\
\text { cession de licences } \\
\text { Acquisition }\end{array}$ & $\begin{array}{l}\text { Coentreprise } \\
\text { (Joint venture) } \\
\text { R\&D sous contrat }\end{array}$ \\
\hline & Stratégique & Non stratégique & Peu familier \\
\hline
\end{tabular}

Figure 3. Les modes d'innovation ouverte selon deux caractéristiques : technologie et marchés [KHE 10]

Cette matrice datant de 2004 fait écho à d'autres travaux, notamment de Sachwald, selon qui, les instruments de l'innovation ouverte sont utilisés à divers niveaux le long du cycle de l'innovation (Figure 4). Dans la phase de recherche et développement, des contrats de sous-traitance peuvent être établis, ainsi que des partenariats publics et/ou privés. L'achat et la cession de licences interviennent plus tardivement. En effet, lorsque les entreprises ont identifié des brevets susceptibles de correspondre à des besoins spécifiques, ou pouvant intéresser d'autres partenaires, l'acquisition et la cession peuvent être réalisées.

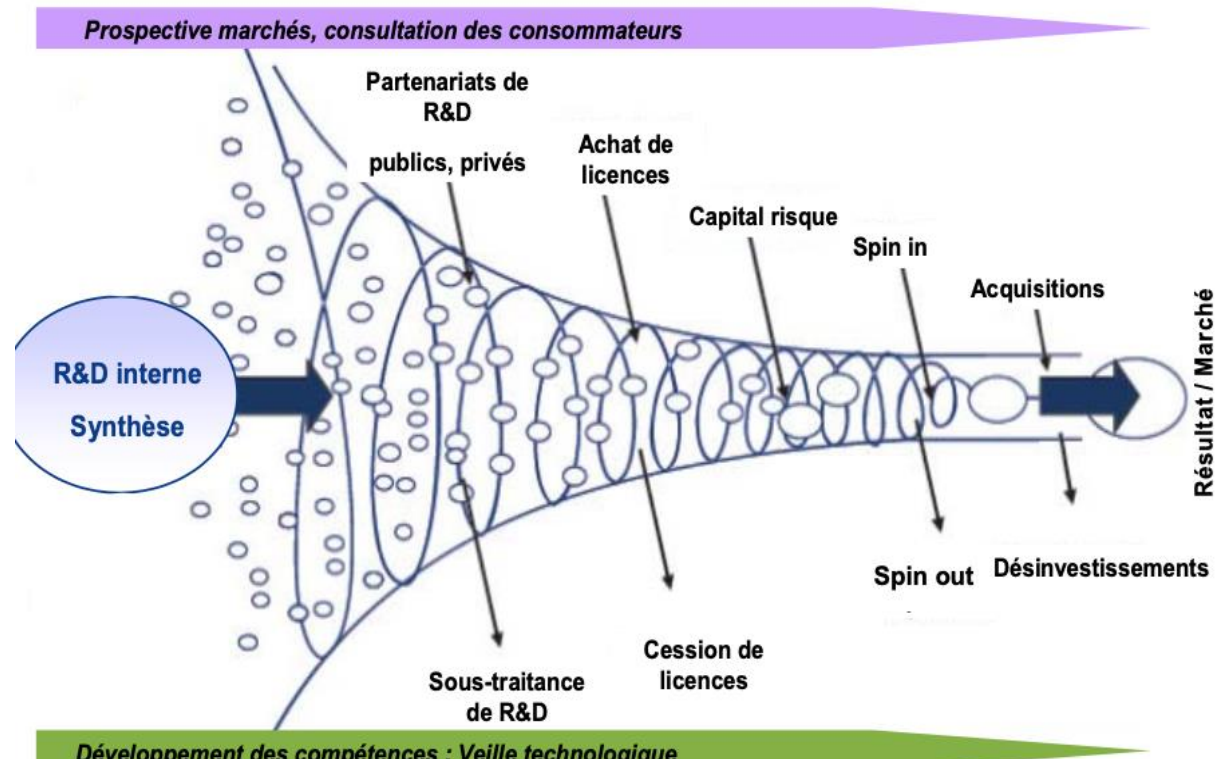

Développement des compétences ; Veille technologique

Figure 4. Les instruments de l'innovation ouverte le long de la chaîne de l'innovation [SAC 08] 
Par ailleurs, la notion de réseau est sous-jacente à celle d'innovation ouverte et innovation collaborative [SUI 18]. Le nombre de liens établis entre acteurs peut être plus important lors des premières étapes du processus de recherche et développement dans le cadre de création des innovations. En effet, établir des liens et tisser un réseau est d'autant plus important lors des phases de recherche d'idées et d'acquisition des connaissances. Se baser sur les complémentarités des acteurs et leurs spécialités est ensuite préconisé pour les phases de développement de l'innovation. De plus, des acquisitions d'entreprises peuvent avoir lieu lorsque les compétences dont elle bénéficie sont primordiales pour l'innovation à développer. Enfin, de manière à optimiser l'implémentation et la diffusion de l'innovation, d'autres types d'acteurs et de partenariats peuvent être sollicités [SAC 08, SUI 18].

Les avantages amenés par un paradigme d'innovation ouverte et prônant le recours à des collaborations ont été longuement étudiés et mis en avant dans beaucoup de travaux. La diversité des partenaires est généralement un atout mis en lumière. Cependant, il est important de noter également des difficultés rencontrées lors de la mise en place de tel processus. En effet, des problèmes de différente nature peuvent survenir, particulièrement celui du langage utilisé. Les entreprises faisant partie de secteurs différents n'utilisent nécessairement pas le même vocabulaire. De ce fait, un langage commun est essentiel pour se faire comprendre et communiquer de manière efficiente. Par ailleurs, la propriété intellectuelle représente un point sensible de l'innovation ouverte. Les savoirs générés peuvent être partagés et révélés à plusieurs partenaires externes qui peuvent alors les utiliser et/ou concurrencer [BUR 13, KHE 10]. Enfin, les problèmes managériaux liés au mode de fonctionnement et la structure organisationnelle des entreprises peuvent survenir, surtout dans des contextes ou petites et moyennes entreprises collaborent avec de plus grandes entreprises où le pouvoir décisionnel est plus hiérarchisé. Les contraintes temporelles et financières font également partie des points de discorde potentiels [BUR 13].

\section{Conclusion}

L'innovation ouverte, l'innovation collaborative et les autres termes utilisés faisant référence aux concepts développés initialement par Henri Chesbrough sont dorénavant très largement répandus dans différentes disciplines, du management à l'économie en passant par les sciences sociales. Plusieurs discussions et critiques restent ouvertes quant à l'aspect d'ouverture que les entreprises devraient réaliser dans les processus d'innovation. Les réseaux d'innovation peuvent, dans ce cadre, être un soutien pour les firmes et un support de lancement d'initiatives innovantes ou de mise en relation avec des acteurs clés, de manière à promouvoir des collaborations amenant à l'innovation.

Par ailleurs, peu de travaux ont étudié la dimension territoriale de l'innovation ouverte [LOI 11]. La proximité physique est garante de la circulation de connaissances et flux productifs entre partenaires, généralement regroupés comme dans les cas des clusters ou districts industriels. Cette forme de proximité est complétée par la proximité cognitive et institutionnelle quand cela est nécessaire. Par ailleurs, dans de telles conceptions organisationnelles, des réseaux peuvent se créer en intégrant des acteurs locaux présents historiquement sur le territoire et de nouveaux acteurs identifiés comme ressources utiles pour un projet. Le recours à des facilitateurs locaux ou intermédiaires (par exemple des centres de recherche locaux) bénéficiant d'une proximité physique indispensable est souvent privilégié, comme c'est le cas dans l'exemple du district industriel de l'Emilie Romagne en Italie. Dans cet exemple, le district a recours à des acteurs aux niveaux national et international, ainsi que les centres de recherche de proximité.

L'innovation collaborative implique le recours à plusieurs formes de partenariats, principalement établi à certaines étapes du processus de création d'innovation en fonction des besoins des acteurs, de la stratégie pour intégrer le marché et des technologies présentes. Néanmoins, la présence de plusieurs partenaires peut engendrer des difficultés, notamment des problèmes de gestion, financiers, de propriété intellectuelle et de langage commun. Ils connectent les acteurs entre eux, ils impliquent, 
engagent et mobilisent les acteurs. Ils ont également un rôle de résoudre, éviter et miter les conflits d'intérêts, et enfin ils stimulent activement le processus

Finalement, les systèmes nationaux d'innovation favorisant la production de technologie, de science, ainsi que de connaissances, leur diffusion et leur utilisation dessine un cadre national dans lequel les entreprises peuvent innover, via des interactions entre secteurs publics et privés [CAS 06]. Par ailleurs, les systèmes régionaux d'innovation ont également leur part dans la promotion de l'innovation collaborative au sein de cadres plus territorialisés et dispatchés, qui sont à l'œuvre dans nombre de territoires européens.

\section{Bibliographie}

[AGO 13] AgOGUÉ M., YstRÖM A., LE MASSON P. « Rethinking the role of intermediaries as an architect of collective exploration and creation of knowledge in open innovation », International Journal of Innovation Management, 17(02), $1-32,2013$.

[AOU 19] AOUINAÏT C., Innovation capacity of the agricultural sector: Network of productive interactions, knowledge transfer and differentiation mechanisms. Thèse de doctorat, École Polytechnique Fédérale de Lausanne, 2019.

[BOS 05] BoschmA, R., « Proximity and Innovation: A Critical Assessment », Regional Studies, 39(1), 61-74, 2005.

[BUR 13] Burger-Helmcehn T., Penin J., GuitTard C., SChenk E., DinTRICh A., L'innovation ouverte : Définition, pratiques et perspectives. Chambre de commerce et d'industrie de Paris, 2013.

[CAS 06] CASAdella V., BenlahCEN-TlemCani M., « De l'applicabilité du Système National d'Innovation dans les Pays Moins Avancés », Innovations, 24(2), 59, 2006.

[CHA 08] ChAMPENOIS C., «La co-localisation d'entreprises innovantes comme non-choix. L'exemple de l'industrie allemande des biotechnologies », Géographie, économie, société, 10(1), 61-86, 2008.

[CHE 03] Chesbrough H. W., Open Innovation: The New Imperative for Creating and Profiting from Technology, Harvard Business School Publishing, Brighton, 2003.

[CHE 06] Chesbrough H. W., Open Business Models: How To Thrive In The New Innovation Landscape, Harvard Business School Publishing, Brighton, 2003.

[CHE 12] Chesbrough H. W., «Open Innovation: Where We've Been and Where We're Going », Research-Technology Management, 55(4), 20-27, 2012.

[DAH 10] DAHLANDER L, GANN D.M., « How open is innovation? », Research Policy, 39(6), 699-709, 2010.

[DAU 07] DAUMAS J-C., « Districts industriels: Du concept à l'histoire : Les termes du débat », Revue économique, 58(1), 131-152, 2007.

[GAS 10] Gassmann O., EnKel E., Chesbrough H.W., « The future of open innovation », R\&D Management, 40(3), 213-221, 2010.

[HOW 06] HowELLS J., « Intermediation and the role of intermediaries in innovation », Research Policy, 35(5), 715-728, 2006.

[INS 08] Institut D’AmENAGEMENT ET D’URbanisme DE LA REgION D’Ile-DE-FranCE, Clusters Mondiaux : Regards croisés sur la théorie et la réalité des clusters. Identification et cartographie des principaux clusters internationaux, en ligne : en ligne : https://halshs.archives-ouvertes.fr/halshs-00412049/documents, 2008.

[ISC 11] ISCHIA T., LESCOP D., "Une analyse critique des fondements de l'innovation ouverte », Revue française de gestion, 37(210), 87-98, 2011.

[KHE 10] KHEDHER S., L'écosystème d'une firme: Une stratégie de gestion de l'innovation ouverte, Thèse de doctorat, Université du Québec à Montréal, 2010.

[KLE 12] KLEWITZ J., ZEYEN A., HANSEN E.G., « Intermediaries driving eco-innovation in SMEs: A qualitative investigation », European Journal of Innovation Management, 15(4), 442-467, 2012.

[LER 14] LeROuX I., Muller P., PlotTU B., WidehEM C., «Innovation ouverte et évolution des business models dans les pôles de compétitivité: Le rôle des intermédiaires dans la création variétale végétale », Revue d'économie industrielle, 146, 115-151, 2014. 
[LOI 11] LOILIER T., TELLIER A., «Que faire du modèle de l'innovation ouverte ? », Revue française de gestion, 37(210), 69-85, 2011.

[MIN 82] MinTZBERG H., Structure et dynamique des organisations, Les Éditions d'Organisation, 1982.

[PEC 02] PeCQUeUR B., ZIMMERMAnN J-B., Les fondements d'une économie de proximité, Hermes-Lavoisier, 2004.

[PON 10] PONCET J., KUPER M., CHICHE J., « Wandering off the paths of planned innovation: The role of formal and informal intermediaries in a large-scale irrigation scheme in Morocco », Agricultural Systems, 103(4), 171-179, 2002.

[POR 90] PORTER M.E., The competitive advantage of nations, Harvard Business Review, 71-91, 1990.

[RAL 04] RALLET A., TORRE A., « Proximité et localisation », Économie rurale, 280(1), 25-41, 2004.

[SAC 08] SACHWALD F., Réseaux mondiaux d'innovation ouverte, systèmes nationaux et politiques publiques, Ministère de l'Enseignement supérieur et de la Recherche, 2008.

[STE 14] STEINER A., «Élaboration et mise en œuvre d'un modèle organisationnel favorisant l'open innovation: Contribution à l'innovation collaborative », Revue Française de Gestion, 71-84, 2014.

[SUI 18] SUIRE R., BERTHINIER-Poncet A., FABBri J., «Les stratégies de l'innovation collective: Communautés, organisations, territoires », Revue Française de Gestion, 44, 71-84, 2018.

[TOR 16] TORRE A. «La figure du réseau : Dimensions spatiales et organisationnelles », Géographie, Économie, Société, $18,455-469,2016$.

[VON 94] VON HIPPEL E., «Sticky Information » and the Locus of Problem Solving: Implications for Innovation », Management Science, 40(4), 429-439, 1994.

[WAN 11] WANG Y., ROIJAKKERS N., VANHAVERBEKE W., « Linking open innovation to national systems of innovation: A coevolutionary perspective », International Journal of Innovation and Regional Development, 3(5), 446, 2011. 